\title{
Rigorous derivation of the mean field Green functions of the two-band Hubbard model of superconductivity
}

\author{
Gh Adam and S Adam \\ Laboratory of Information Technologies, Joint Institute for Nuclear Research, \\ 141980 Dubna, Moscow Region, Russia \\ and \\ Horia Hulubei National Institute for Physics and Nuclear Engineering (IFIN-HH), \\ 407 Atomistilor, Magurele-Bucharest, 077125 Romania
}

E-mail: adamg@jinr.ru, adams@jinr.ru

\begin{abstract}
The Green function (GF) equation of motion technique for solving the effective two-band Hubbard model of high- $T_{c}$ superconductivity in cuprates [N.M. Plakida et al., Phys. Rev. B, 51, 16599 (1995); JETP, 97, 331 (2003)] rests on the Hubbard operator (HO) algebra. We show that, if we take into account the invariance to translations and spin reversal, the $\mathrm{HO}$ algebra results in invariance properties of several specific correlation functions. The use of these properties allows rigorous derivation and simplification of the expressions of the frequency matrix (FM) and of the generalized mean field approximation (GMFA) Green functions (GFs) of the model.

For the normal singlet hopping and anomalous exchange pairing correlation functions which enter the FM and GMFA-GFs, an approximation procedure based on the identification and elimination of exponentially small quantities is described. It secures the reduction of the correlation order to GMFA-GF expressions.

PACS numbers: 74.20.-z, 74.20.Mn, 74.72.-h
\end{abstract}

Submitted to: J. Phys. A: Math. Gen. 


\section{Introduction}

A consistent theoretical model of the high critical temperature superconductivity in cuprates is to be able to accommodate both the normal and superconducting states under incorporation of the essential features of these systems (see, e.g., [1] for a review): strong antiferromagnetic (AFM) superexchange interaction inside the $\mathrm{CuO}_{2}$ planes, occurrence of two relatively isolated energy bands around the Fermi level, able to develop $d_{x^{2}-y^{2}}$ pairing: one stemming from single particle copper $d_{x^{2}-y^{2}}$ states and the second one from singlet doubly occupied states generated [2] by crystal field interaction; hopping conduction for an extremely low density of the free charge carriers.

The $p$ - $d$ model [3], while incorporating all these features, is too cumbersome and cell-cluster perturbation theory [4, 5] providing a hierarchy of the various interaction terms was used to derive simpler models from it. Extreme limit cases of this reduction procedure are various effective one-band $t$ - $J$ models (see, e.g., [6, 7] and references therein) which, while unveiling the role played by the AFM exchange interaction in the occurrence of the $d$-wave pairing, address exclusively the superconducting state.

The reduction of the $p-d$ model to an effective two-band Hubbard model considered by Plakida et al. 8], corroborated with the use of the equation of motion technique for thermodynamic Green functions (GF) [9], provided the simplest approach to the description of both the normal [8, 10] and the superconducting states [11, 12, 13] within a frame securing rigorous fulfilment of the Pauli exclusion principle for fermionic states.

The Green function technique rests on the Hubbard operator algebra. Its rigorous implementation onto a system characterized by specific symmetry properties (translation invariant two-dimensional spin lattice, spin reversal invariance of the observables) results either in characteristic invariance properties of several correlation functions, or in the occurrence of some exactly vanishing correlation functions. The use of these results allows rigorous derivation and simplification of the expressions of the frequency matrix and of the generalized mean field approximation (GMFA) Green functions of the model.

The obtained expressions contain higher order boson-boson correlation functions (CFs). For the CFs involving singlets (normal singlet hopping CFs and anomalous exchange pairing $\mathrm{CFs}$ ), an approximation procedure which avoids the usual decoupling schemes and, yet, secures the correlation order reduction to GMFA-GF expressions, under the identification and elimination of exponentially small quantities, is described.

The organization of the paper is as follows. Sec. 2 summarizes essentials of the two-band Hubbard model and GMFA-GF equations. Sec. 3 describes the invariance properties following from the translation invariance of the underlying spin lattice. Sec. [4 derives invariance properties and constraints following from the invariance of the macroscopic properties of the system under spin reversal. On the basis of the results of Sec. 3 and 4 , rigorous derivation of the frequency matrix in the $(\mathbf{r}, \omega)$-representation is done in Sec. [5. The derivation of GMFA-GF expressions for the boson-boson correlation functions involving singlets is discussed in Sec. 6 .

Collecting together the results of sections 5 and 6 , expressions of the frequency 
matrix and of the GMFA Green function matrix are derived in the $(\mathbf{q}, \omega)$-representation in sections 7 and 8 respectively. These results explicitly incorporate both hole-doping and electron-doping features of the cuprate systems through the singlet hopping and superconducting pairing terms.

The paper ends with conclusions in section 9 .

\section{Mean field approximation}

The Hamiltonian of the effective two-band singlet-hole Hubbard model $[8]$ is written in the form

$$
\begin{aligned}
H & =E_{1} \sum_{i, \sigma} X_{i}^{\sigma \sigma}+E_{2} \sum_{i} X_{i}^{22}+ \\
& +\mathcal{K}_{11} \sum_{i, \sigma} \tau_{1, i}^{\sigma 0,0 \sigma}+\mathcal{K}_{22} \sum_{i, \sigma} \tau_{1, i}^{2 \sigma, \sigma 2}+\mathcal{K}_{21} \sum_{i, \sigma} 2 \sigma\left(\tau_{1, i}^{2 \bar{\sigma}, 0 \sigma}+\tau_{1, i}^{\sigma 0, \bar{\sigma} 2}\right)
\end{aligned}
$$

The summation label $i$ runs over the sites of an infinite two-dimensional (2D) square array the lattice constants of which, $a_{x}=a_{y}$, are defined by the underlying single crystal structure. The spin projection values in the sums over $\sigma$ are $\sigma= \pm 1 / 2, \bar{\sigma}=-\sigma$.

The Hubbard operators (HOs) $X_{i}^{\alpha \beta}=|i \alpha\rangle\langle i \beta|$ are defined for the four states of the model at each lattice site $i$ : $|0\rangle$ (vacuum), $|\sigma\rangle=|\uparrow\rangle$ and $|\bar{\sigma}\rangle=|\downarrow\rangle$ (single particle spin states inside the hole subband), and $|2\rangle=|\uparrow \downarrow\rangle$ (singlet state in the singlet subband).

The multiplication rule holds $X_{i}^{\alpha \beta} X_{i}^{\gamma \eta}=\delta_{\beta \gamma} X_{i}^{\alpha \eta}$. The HOs may be fermionic (single spin state creation/annihilation in a subband) or bosonic (singlet creation/annihilation, spin or charge densities, particle numbers). For a pair of fermionic HOs, the anticommutator rule holds $\left\{X_{i}^{\alpha \beta}, X_{j}^{\gamma \eta}\right\}=\delta_{i j}\left(\delta_{\beta \gamma} X_{i}^{\alpha \eta}+\delta_{\eta \alpha} X_{i}^{\gamma \beta}\right)$ whereas, if one or both HOs are bosonic, the commutation rule holds $\left[X_{i}^{\alpha \beta}, X_{j}^{\gamma \eta}\right]=\delta_{i j}\left(\delta_{\beta \gamma} X_{i}^{\alpha \eta}-\delta_{\eta \alpha} X_{i}^{\gamma \beta}\right)$. At each lattice site $i$, the constraint of no double occupancy of any quantum state $|i \alpha\rangle$ is rigorously fulfilled due to the completeness relation $X_{i}^{00}+X_{i}^{\sigma \sigma}+X_{i}^{\bar{\sigma} \bar{\sigma}}+X_{i}^{22}=1$.

In (11), $E_{1}=\tilde{\varepsilon_{d}}-\mu$ denotes the hole subband energy for the renormalized energy $\tilde{\varepsilon_{d}}$ of a $d$-hole and the chemical potential $\mu$. The energy parameter of the singlet subband is $E_{2}=2 E_{1}+\Delta$, where $\Delta \approx \Delta_{p d}=\varepsilon_{p}-\varepsilon_{d}$ is an effective Coulomb energy $U_{\text {eff }}$ corresponding to the difference between the two energy levels of the model.

In the description of the hopping processes, the label 1 points to the hole subband and 2 to the singlet subband. The hopping energy parameter $\mathcal{K}_{a b}=2 t_{p d} K_{a b}$ depends on $t_{p d}$, the hopping $p$ - $d$ integral, and on energy band dependent form factors, $K_{a b}$. Inband $\left(\mathcal{K}_{11}, \mathcal{K}_{22}\right)$ and interband $\left(\mathcal{K}_{21}=\mathcal{K}_{12}\right)$ processess are present. The Hubbard 1-forms

$$
\tau_{1, i}^{\alpha \beta, \gamma \eta}=\sum_{m \neq i} \nu_{i m} X_{i}^{\alpha \beta} X_{m}^{\gamma \eta}
$$

incorporate the overall effects of specific hopping processes (through the labels $(\alpha \beta, \gamma \eta)$ of the pair of Hubbard operators) involving the lattice site $i$ and its neighbouring sites.

Up to three coordination spheres around the reference site $i$ do contribute [4, 5] to the sum (2), each being characterized by a small specific value of the overlap coefficients 
$\nu_{i j}\left(\nu_{1}\right.$ for the nearest neighbour $(\mathrm{nn}), \nu_{2}$ for the next nearest neighbour (nnn), $\nu_{3}$ for the third coordination spheres).

The quasi-particle spectrum and superconducting pairing for the Hamiltonian (1) are obtained [11, 12] from the two-time $4 \times 4$ GF matrix (in Zubarev notation [9])

$$
\tilde{G}_{i j \sigma}\left(t-t^{\prime}\right)=\left\langle\left\langle\hat{X}_{i \sigma}(t) \mid \hat{X}_{j \sigma}^{\dagger}\left(t^{\prime}\right)\right\rangle\right\rangle=-\mathrm{i} \theta\left(t-t^{\prime}\right)\left\langle\left\{\hat{X}_{i \sigma}(t), \hat{X}_{j \sigma}^{\dagger}\right\}\right\rangle,
$$

where $\langle\cdots\rangle$ denotes the statistical average over the Gibbs grand canonical ensemble.

The GF (3) is defined for the four-component Nambu column operator

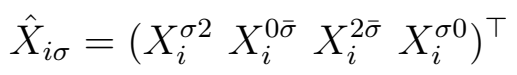

where the superscript $\top$ denotes the transposition. In (3),$\hat{X}_{j \sigma}^{\dagger}=\left(X_{j}^{2 \sigma} X_{j}^{\bar{\sigma} 0} X_{j}^{\bar{\sigma} 2} X_{j}^{0 \sigma}\right)$ is the adjoint operator of $\hat{X}_{j \sigma}$.

The GF matrix in $(\mathbf{r}, \omega)$-representation is related to the expression (3) of the GF matrix in $(\mathbf{r}, t)$-representation by the non-unitary Fourier transform,

$$
\tilde{G}_{i j \sigma}\left(t-t^{\prime}\right)=\frac{1}{2 \pi} \int_{-\infty}^{+\infty} \tilde{G}_{i j \sigma}(\omega) \mathrm{e}^{-\mathrm{i} \omega\left(t-t^{\prime}\right)} \mathrm{d} \omega .
$$

The energy spectrum of the translation invariant spin lattice of (1) is solved in the reciprocal space. The GF matrix in this $(\mathbf{q}, \omega)$-representation is related to the GF matrix in $(\mathbf{r}, \omega)$-representation by the non-unitary discrete Fourier transform

$$
\tilde{G}_{i j \sigma}(\omega)=\frac{1}{N} \sum_{\mathbf{q}} \mathrm{e}^{-\mathrm{iq}\left(\mathbf{r}_{j}-\mathbf{r}_{i}\right)} \tilde{G}_{\sigma}(\mathbf{q}, \omega) .
$$

For an elemental GF of labels $(\alpha \beta, \gamma \eta)$, we use the notation $\left\langle\left\langle X_{i}^{\alpha \beta}(t) \mid X_{j}^{\gamma \eta}\left(t^{\prime}\right)\right\rangle\right\rangle$ in the $(\mathbf{r}, t)$-representation and, similarly, $\left\langle\left\langle X_{i}^{\alpha \beta} \mid X_{j}^{\gamma \eta}\right\rangle\right\rangle_{\omega}$ (assuming Hubbard operators at $t=0)$, in the $(\mathbf{r}, \omega)$-representation. In the $(\mathbf{q}, \omega)$-representation, it is convenient to use the notation $G^{\alpha \beta, \gamma \eta}(\mathbf{q}, \omega)$.

We shall consider henceforth the GMFA-GF, $\tilde{G}_{\sigma}^{0}(\mathbf{q}, \omega)$. Its derivation involves:

(i) Differentiation of the GF (3) with respect to $t$ and use of the equations of motion for the Heisenberg operators $X_{i}^{\alpha \beta}(t)$.

(ii) Derivation of an algebraic equation for $\tilde{G}_{i j \sigma}(\omega)$, Eq. (15).

(iii) Elimination of the contribution of the inelastic processes to the commutator $\hat{Z}_{i \sigma}=\left[\hat{X}_{i \sigma}, H\right]$ entering the equation of motion of $\tilde{G}_{i j \sigma}(\omega)$.

(iv) Transformation to $(\mathbf{q}, \omega)$-representation of the obtained equation of $\tilde{G}_{i j \sigma}^{0}(\omega)$ by means of the Fourier transform (6).

This finally yields

$$
\begin{aligned}
& \tilde{G}_{\sigma}^{0}(\mathbf{q}, \omega)=\tilde{\chi}\left[\tilde{\chi} \omega-\tilde{\mathcal{A}}_{\sigma}(\mathbf{q})\right]^{-1} \tilde{\chi}, \\
& \tilde{\chi}=\left\langle\left\{\hat{X}_{i \sigma}, \hat{X}_{i \sigma}^{\dagger}\right\}\right\rangle, \\
& \tilde{\mathcal{A}}_{\sigma}(\mathbf{q})=\sum_{\mathbf{r}_{i j}} \mathrm{e}^{\mathbf{i q}\left(\mathbf{r}_{j}-\mathbf{r}_{i}\right)} \tilde{\mathcal{A}}_{i j \sigma}, \quad \mathbf{r}_{i j}=\mathbf{r}_{j}-\mathbf{r}_{i}, \\
& \tilde{\mathcal{A}}_{i j \sigma}=\left\langle\left\{\left[\hat{X}_{i \sigma}, H\right], \hat{X}_{j \sigma}^{\dagger}\right\}\right\rangle .
\end{aligned}
$$

The matrix $\tilde{\mathcal{A}}_{i j \sigma}$ is Hermitian. 


\section{Translation invariance of the spin lattice}

Four consequences follow from the translation invariance of the spin lattice.

- The definition of the Hubbard 1-form (2) over a translation invariant spin lattice results in the identity (which secures the hermiticity of the Hamiltonian $H$ ):

$$
\tau_{1, i}^{\alpha \beta, \gamma \eta}=-\tau_{1, i}^{\gamma \eta, \alpha \beta}
$$

- The Green function (3) of the model Hamiltonian (1) depends only on the distance $r_{i j}=\left|\mathbf{r}_{j}-\mathbf{r}_{i}\right|$ between the position vectors at the lattice sites $i$ and $j[9]$.

- The one-site statistical averages are independent on the site label $i,\left\langle X_{i}^{\alpha \beta}\right\rangle=\left\langle X_{j}^{\alpha \beta}\right\rangle$, $(\forall i, j)$. For this reason, the site label in the one-site averages will be omitted.

- The two-site statistical averages $\left\langle X_{i}^{\alpha \beta} X_{j}^{\gamma \eta}\right\rangle$ remain invariant under the interchange of the site labels $i$ and $j$,

$$
\left\langle X_{i}^{\alpha \beta} X_{j}^{\gamma \eta}\right\rangle=\left\langle X_{j}^{\alpha \beta} X_{i}^{\gamma \eta}\right\rangle, \quad i \neq j
$$

\section{Spin reversal invariance}

The energy spectrum of the system described by the Hamiltonian (1) does not depend on the specific values $\sigma= \pm 1 / 2$ of the spin projection. As a consequence, the definition of the GF (3) either in terms of the $\sigma$-Nambu operator (4) or the $\bar{\sigma}$-Nambu operator

$$
\hat{X}_{i \bar{\sigma}}=\left(X_{i}^{\bar{\sigma} 2} X_{i}^{0 \sigma} X_{i}^{2 \sigma} X_{i}^{\bar{\sigma} 0}\right)^{\top}
$$

has to result in mathematically equivalent descriptions of the observables. This means, however, that the mathematical structures of the frequency matrices $\tilde{\mathcal{A}}_{i j \sigma}$, Eq. (10), and $\tilde{\mathcal{A}}_{i j \bar{\sigma}}=\left\langle\left\{\left[\hat{X}_{i \bar{\sigma}}, H\right], \hat{X}_{j \bar{\sigma}}^{\dagger}\right\}\right\rangle$ emerging from the $\bar{\sigma}$-Nambu operator (13), have to be related to each other.

The identification of the existing relationships is constructive: we calculate and compare the corresponding matrix elements of $\tilde{\mathcal{A}}_{i j \sigma}$ and $\tilde{\mathcal{A}}_{i j \bar{\sigma}}$. The multiplication rules and the commutation/anticommutation relations satisfied by the Hubbard operators result in the following general expression of the elemental anticommutators entering their definitions:

$$
\left\{\left[X_{i}^{\lambda \mu}, H\right], X_{j}^{\nu \varphi}\right\}=\delta_{i j} C_{i}^{\lambda \mu, \nu \varphi}+\left(1-\delta_{i j}\right) \nu_{i j} T_{i j}^{\lambda \mu, \nu \varphi},
$$

with one-site contributions given by

$$
\begin{aligned}
C_{i}^{\lambda \mu, \nu \varphi}=\delta_{\nu \mu}\{ & {\left[\left(\sum_{\sigma} \delta_{\mu \sigma}\right) E_{1}+\delta_{\mu 2} E_{2}\right] X_{i}^{\lambda \varphi}+} \\
& +\sum_{\sigma} \delta_{\lambda \sigma}\left[-E_{1} X_{i}^{\sigma \varphi}+\mathcal{K}_{11} \tau_{1, i}^{0 \varphi, \sigma 0}-\mathcal{K}_{22} \tau_{1, i}^{2 \varphi, \sigma 2}+\mathcal{K}_{21} \cdot 2 \sigma\left(\tau_{1, i}^{2 \varphi, 0 \bar{\sigma}}+\tau_{1, i}^{0 \varphi, 2 \bar{\sigma}}\right)\right]+ \\
& +\delta_{\lambda 2}\left(-E_{2} X_{i}^{2 \varphi}+\mathcal{K}_{22} \sum_{\sigma} \tau_{1, i}^{\sigma \varphi, 2 \sigma}+\mathcal{K}_{21} \sum_{\sigma} 2 \sigma \tau_{1, i}^{\bar{\sigma} \varphi, \sigma 0}\right)- \\
& \left.-\delta_{\lambda 0}\left(\mathcal{K}_{11} \sum_{\sigma} \tau_{1, i}^{\sigma \varphi, 0 \sigma}+\mathcal{K}_{21} \sum_{\sigma} 2 \sigma \tau_{1, i}^{\sigma \varphi, \bar{\sigma} 2}\right)\right\}+
\end{aligned}
$$


Mean field Green functions of Hubbard model of superconductivity

$$
\begin{aligned}
&+\delta_{\varphi \lambda}\{-\left[\left(\sum_{\sigma} \delta_{\lambda \sigma}\right) E_{1}+\delta_{\lambda 2} E_{2}\right] X_{i}^{\nu \mu}+ \\
&+\sum_{\sigma} \delta_{\mu \sigma}\left[E_{1} X_{i}^{\nu \sigma}+\mathcal{K}_{11} \tau_{1, i}^{\nu 0,0 \sigma}-\mathcal{K}_{22} \tau_{1, i}^{\nu 2,2 \sigma}+\mathcal{K}_{21} \cdot 2 \sigma\left(\tau_{1, i}^{\nu 2, \bar{\sigma} 0}+\tau_{1, i}^{\nu 0, \bar{\sigma} 2}\right)\right]+ \\
&+\delta_{\mu 2}\left(E_{2} X_{i}^{\nu 2}+\mathcal{K}_{22} \sum_{\sigma} \tau_{1, i}^{\nu \sigma, \sigma 2}+\mathcal{K}_{21} \sum_{\sigma} 2 \sigma \tau_{1, i}^{\nu \bar{\sigma}, 0 \sigma}\right)- \\
&\left.-\delta_{\mu 0}\left(\mathcal{K}_{11} \sum_{\sigma} \tau_{1, i}^{\nu \sigma, \sigma 0}+\mathcal{K}_{21} \sum_{\sigma} 2 \sigma \tau_{1, i}^{\nu \sigma, 2 \bar{\sigma}}\right)\right\}- \\
&- \sum_{\sigma} \delta_{\lambda \sigma}\left[\delta_{\varphi 0}\left(\mathcal{K}_{11} \tau_{1, i}^{\nu \mu, \sigma 0}+2 \sigma \mathcal{K}_{21} \tau_{1, i}^{\nu \mu, 2 \bar{\sigma}}\right)-\delta_{\varphi 2}\left(\mathcal{K}_{22} \tau_{1, i}^{\nu \mu, \sigma 2}-2 \sigma \mathcal{K}_{21} \tau_{1, i}^{\nu \mu, 0 \bar{\sigma}}\right)\right]+ \\
&+\sum_{\sigma} \delta_{\varphi \sigma}\left[\delta_{\lambda 0}\left(\mathcal{K}_{11} \tau_{1, i}^{\nu \mu, 0 \sigma}+2 \sigma \mathcal{K}_{21} \tau_{1, i}^{\nu \mu, \bar{\sigma} 2}\right)-\delta_{\lambda 2}\left(\mathcal{K}_{22} \tau_{1, i}^{\nu \mu, 2 \sigma}-2 \sigma \mathcal{K}_{21} \tau_{1, i}^{\nu \mu, \bar{\sigma} 0}\right)\right]- \\
&-\sum_{\sigma} \delta_{\mu \sigma}\left[\delta_{\nu 0}\left(\mathcal{K}_{11} \tau_{1, i}^{\lambda \varphi, 0 \sigma}+2 \sigma \mathcal{K}_{21} \tau_{1, i}^{\lambda \varphi, \bar{\sigma} 2}\right)-\delta_{\nu 2}\left(\mathcal{K}_{22} \tau_{1, i}^{\lambda \varphi, 2 \sigma}-2 \sigma \mathcal{K}_{21} \tau_{1, i}^{\lambda \varphi, \bar{\sigma} 0}\right)\right]+ \\
&+\sum_{\sigma} \delta_{\nu \sigma}\left[\delta_{\mu 0}\left(\mathcal{K}_{11} \tau_{1, i}^{\lambda \varphi, \sigma 0}+2 \sigma \mathcal{K}_{21} \tau_{1, i}^{\lambda \varphi, 2 \bar{\sigma}}\right)-\delta_{\mu 2}\left(\mathcal{K}_{22} \tau_{1, i}^{\lambda \varphi, \sigma 2}-2 \sigma \mathcal{K}_{21} \tau_{1, i}^{\lambda \varphi, 0 \bar{\sigma}}\right)\right]
\end{aligned}
$$

and two-site contributions given by

$$
\begin{aligned}
T_{i j}^{\lambda \mu, \nu \varphi}= & \delta_{\nu \mu}\left[\left(\sum_{\sigma} \delta_{\mu \sigma}\right)\left(\mathcal{K}_{11} X_{i}^{\lambda 0} X_{j}^{0 \varphi}-\mathcal{K}_{22} X_{i}^{\lambda 2} X_{j}^{2 \varphi}\right)+\left(-\delta_{\mu 0} \mathcal{K}_{11}+\delta_{\mu 2} \mathcal{K}_{22}\right) \sum_{\sigma} X_{i}^{\lambda \sigma} X_{j}^{\sigma \varphi}\right]+ \\
+ & \delta_{\varphi \lambda}\left[\left(\sum_{\sigma} \delta_{\lambda \sigma}\right)\left(-\mathcal{K}_{11} X_{i}^{0 \mu} X_{j}^{\nu 0}+\mathcal{K}_{22} X_{i}^{2 \mu} X_{j}^{\nu 2}\right)+\left(\delta_{\lambda 0} \mathcal{K}_{11}-\delta_{\lambda 2} \mathcal{K}_{22}\right) \sum_{\sigma} X_{i}^{\sigma \mu} X_{j}^{\nu \sigma}\right]- \\
& -\sum_{\sigma} \delta_{\lambda \sigma}\left\{\delta_{\nu 0} \mathcal{K}_{11} X_{i}^{0 \mu} X_{j}^{\sigma \varphi}-\delta_{\nu 2} \mathcal{K}_{22} X_{i}^{2 \mu} X_{j}^{\sigma \varphi}+\right. \\
& \left.+\mathcal{K}_{21} \cdot 2 \sigma\left[\delta_{\varphi 0} X_{i}^{2 \mu} X_{j}^{\nu \bar{\sigma}}+\delta_{\varphi 2} X_{i}^{0 \mu} X_{j}^{\nu \bar{\sigma}}+\delta_{\nu,-\lambda}\left(X_{i}^{2 \mu} X_{j}^{0 \varphi}+X_{i}^{0 \mu} X_{j}^{2 \varphi}\right)\right]\right\}+ \\
+ & \sum_{\sigma} \delta_{\mu \sigma}\left\{\delta_{\varphi 0} \mathcal{K}_{11} X_{i}^{\lambda 0} X_{j}^{\nu \sigma}-\delta_{\varphi 2} \mathcal{K}_{22} X_{i}^{\lambda 2} X_{j}^{\nu \sigma}+\right. \\
& \left.+\mathcal{K}_{21} \cdot 2 \sigma\left[\delta_{\nu 0} X_{i}^{\lambda 2} X_{j}^{\bar{\sigma} \varphi}+\delta_{\nu 2} X_{i}^{\lambda 0} X_{j}^{\bar{\sigma} \varphi}+\delta_{\varphi,-\mu}\left(X_{i}^{\lambda 2} X_{j}^{\nu 0}+X_{i}^{\lambda 0} X_{j}^{\nu 2}\right)\right]\right\}+ \\
+ & \sum_{\sigma} \delta_{\nu \sigma}\left[\delta_{\lambda 0} \mathcal{K}_{11} X_{i}^{\sigma \mu} X_{j}^{0 \varphi}-\delta_{\lambda 2} \mathcal{K}_{22} X_{i}^{\sigma \mu} X_{j}^{2 \varphi}+\mathcal{K}_{21} \cdot 2 \sigma\left(\delta_{\mu 0} X_{i}^{\lambda \bar{\sigma}} X_{j}^{2 \varphi}+\delta_{\mu 2} X_{i}^{\lambda \bar{\sigma}} X_{j}^{0 \varphi}\right)\right]- \\
& -\sum_{\sigma} \delta_{\varphi \sigma}\left[\delta_{\mu 0} \mathcal{K}_{11} X_{i}^{\lambda \sigma} X_{j}^{\nu 0}-\delta_{\mu 2} \mathcal{K}_{22} X_{i}^{\lambda \sigma} X_{j}^{\nu 2}+\mathcal{K}_{21} \cdot 2 \sigma\left(\delta_{\lambda 0} X_{i}^{\bar{\sigma} \mu} X_{j}^{\nu 2}+\delta_{\lambda 2} X_{i}^{\bar{\sigma} \mu} X_{j}^{\nu 0}\right)\right]+ \\
+ & +\mathcal{K}_{21} \sum_{\sigma} 2 \sigma\left(\delta_{\lambda 0} \delta_{\nu 2} X_{i}^{\sigma \mu} X_{j}^{\bar{\sigma} \varphi}-\delta_{\lambda 2} \delta_{\nu 0} X_{i}^{\bar{\sigma} \mu} X_{j}^{\sigma \varphi}-\delta_{\mu 0} \delta_{\varphi 2} X_{i}^{\lambda \sigma} X_{j}^{\nu \bar{\sigma}}+\delta_{\mu 2} \delta_{\varphi 0} X_{i}^{\lambda \bar{\sigma}} X_{j}^{\nu \sigma}\right) .
\end{aligned}
$$

The comparison of the results obtained from (14) for the corresponding matrix elements of $\tilde{\mathcal{A}}_{i j \sigma}$ and $\tilde{\mathcal{A}}_{i j \bar{\sigma}}$ and the use of the translation invariance properties (11) and (12) result in four distinct kinds of relationships:

- Under the spin reversal $\sigma \rightarrow \bar{\sigma}$, the following invariance properties hold for the normal one-site statistical averages:

$$
\left\langle X_{i}^{\sigma \sigma}\right\rangle=\left\langle X_{i}^{\bar{\sigma} \bar{\sigma}}\right\rangle
$$




$$
\begin{aligned}
& \left\langle\tau_{1, i}^{\sigma 2,2 \sigma}\right\rangle=\left\langle\tau_{1, i}^{\bar{\sigma} 2,2 \bar{\sigma}}\right\rangle, \quad\left\langle\tau_{1, i}^{0 \bar{\sigma}, \bar{\sigma} 0}\right\rangle=\left\langle\tau_{1, i}^{0 \sigma, \sigma 0}\right\rangle \\
& 2 \sigma\left\langle\tau_{1, i}^{\sigma 2, \bar{\sigma} 0}\right\rangle=2 \bar{\sigma}\left\langle\tau_{1, i}^{\bar{\sigma} 2, \sigma 0}\right\rangle
\end{aligned}
$$

- The identity $\left\langle C_{i}^{\sigma 2,0 \sigma}+C_{i}^{0 \bar{\sigma}, \bar{\sigma} 2}\right\rangle=0$ holds, therefrom we get for the one-site anomalous averages,

$$
\begin{aligned}
& \left\langle X_{i}^{02}\right\rangle=0 \\
& \left\langle\tau_{1, i}^{0 \bar{\sigma}, \bar{\sigma} 2}\right\rangle=-\left\langle\tau_{1, i}^{0 \sigma, \sigma 2}\right\rangle \\
& \left\langle\tau_{1, i}^{0 \bar{\sigma}, 0 \sigma}\right\rangle=\left\langle\tau_{1, i}^{\sigma 2, \bar{\sigma} 2}\right\rangle
\end{aligned}
$$

The first two equations imply that the contributions of the one-site terms $\left\langle X_{i}^{02}\right\rangle$ and $\sum_{\sigma}\left\langle\tau_{1, i}^{0 \bar{\sigma}, \bar{\sigma} 2}\right\rangle$ to the superconducting pairing vanish identically irrespective of the model details (like, e.g., the relationship between the lattice constants $a_{x}$ and $a_{y}$ ). For a rectangular spin lattice $\left(a_{x} \neq a_{y}\right)$, Eq. (20) points to the occurrence of a small non-vanishing one-site contribution to the superconducting pairing originating equally in both energy subbands. However, over the square spin lattice (1) $\left(a_{x}=a_{y}\right)$, each term of (20) vanishes for $d$-wave pairing due to the symmetry in the reciprocal space [12].

- Under the spin reversal $\sigma \rightarrow \bar{\sigma}$, the following invariance properties hold for the two-site statistical averages:

$$
\begin{aligned}
\left\langle X_{i}^{\sigma \sigma} X_{j}^{\sigma \sigma}\right\rangle & =\left\langle X_{i}^{\bar{\sigma} \bar{\sigma}} X_{j}^{\bar{\sigma} \bar{\sigma}}\right\rangle, \quad\left\langle X_{i}^{\sigma \sigma} X_{j}^{\bar{\sigma} \bar{\sigma}}\right\rangle=\left\langle X_{i}^{\bar{\sigma} \bar{\sigma}} X_{j}^{\sigma \sigma}\right\rangle \\
\left\langle X_{i}^{22} X_{j}^{\sigma \sigma}\right\rangle & =\left\langle X_{i}^{22} X_{j}^{\bar{\sigma} \bar{\sigma}}\right\rangle, \quad\left\langle X_{i}^{00} X_{j}^{\sigma \sigma}\right\rangle=\left\langle X_{i}^{00} X_{j}^{\bar{\sigma} \bar{\sigma}}\right\rangle \\
\left\langle X_{i}^{02} X_{j}^{\sigma \sigma}\right\rangle & =\left\langle X_{i}^{02} X_{j}^{\bar{\sigma} \bar{\sigma}}\right\rangle .
\end{aligned}
$$

- The operator of the number of particles at site $i$ within the singlet subband, $N_{i}$, is the sum of spin $\sigma$ and $\bar{\sigma}$ components,

$$
N_{i}=n_{i \sigma}+n_{i \bar{\sigma}}, \quad n_{i \sigma}=X_{i}^{\bar{\sigma} \bar{\sigma}}+X_{i}^{22}, \quad n_{i \bar{\sigma}}=X_{i}^{\sigma \sigma}+X_{i}^{22} .
$$

Similar relationships hold for the number of particles at site $i$ within the hole subband, $N_{i}^{h}$

$$
N_{i}^{h}=n_{i \sigma}^{h}+n_{i \bar{\sigma}}^{h}, \quad n_{i \sigma}^{h}=X_{i}^{\sigma \sigma}+X_{i}^{00}, \quad n_{i \bar{\sigma}}^{h}=X_{i}^{\bar{\sigma} \bar{\sigma}}+X_{i}^{00} .
$$

Due to the completeness relation,

$$
N_{i}+N_{i}^{h}=2, \quad n_{i \sigma}+n_{i \sigma}^{h}=n_{i \bar{\sigma}}+n_{i \bar{\sigma}}^{h}=1 .
$$

These equalities simply reflect the fact that, at a given lattice site $i$, there is a single spin state of predefined spin projection, whereas the total number of spin states equals two.

Therefore, the operator $N_{i}$, Eq. (24), provides unique characterization of the occupied states within the model [8, 12, 10]. 


\section{Frequency matrix in $(r, \omega)$-representation}

A straightforward consequence of the results established in section 4 is the simplest general expression of the frequency matrix $\tilde{\mathcal{A}}_{i j \sigma}$, Eq. (10):

$$
\tilde{\mathcal{A}}_{i j \sigma}=\delta_{i j}\left(\begin{array}{cc}
\hat{c}_{\sigma} & \hat{0} \\
\hat{0} & -\left(\hat{c}_{\bar{\sigma}}\right)^{\top}
\end{array}\right)+\left(1-\delta_{i j}\right)\left(\begin{array}{cc}
\hat{D}_{i j \sigma} & \hat{\Delta}_{i j \sigma} \\
\left(\hat{\Delta}_{i j \sigma}\right)^{\dagger} & -\left(\hat{D}_{i j \bar{\sigma}}\right)^{\top}
\end{array}\right) .
$$

The one-site $2 \times 2$ matrix $\hat{c}_{\sigma}$ is Hermitian, its elements do not depend on the particular lattice site $i$,

$$
\hat{c}_{\sigma}=\left(\begin{array}{cc}
\left(E_{1}+\Delta\right) \chi_{2}+a_{22} & 2 \sigma a_{21} \\
2 \sigma a_{21}^{*} & E_{1} \chi_{1}+a_{22}
\end{array}\right),
$$

and are expressed in terms of the spin reversal invariant quantities

$$
\begin{aligned}
& \chi_{2}=\left\langle n_{i \sigma}\right\rangle=\left\langle n_{i \bar{\sigma}}\right\rangle \\
& \chi_{1}=\left\langle n_{i \sigma}^{h}\right\rangle=\left\langle n_{i \bar{\sigma}}^{h}\right\rangle=1-\chi_{2} \\
& a_{22}=\mathcal{K}_{11}\left\langle\tau_{1}^{0 \bar{\sigma}, \bar{\sigma} 0}\right\rangle-\mathcal{K}_{22}\left\langle\tau_{1}^{\sigma 2,2 \sigma}\right\rangle \\
& a_{21}=\left(\mathcal{K}_{11}-\mathcal{K}_{22}\right) \cdot 2 \sigma\left\langle\tau_{1}^{\sigma 2, \bar{\sigma} 0}\right\rangle+\mathcal{K}_{21}\left(\left\langle\tau_{1}^{0 \bar{\sigma}, \bar{\sigma} 0}\right\rangle-\left\langle\tau_{1}^{\sigma 2,2 \sigma}\right\rangle\right) .
\end{aligned}
$$

The normal hopping $2 \times 2$ matrix $\hat{D}_{i j \sigma}$ is symmetric,

$$
\hat{D}_{i j \sigma}=\left(\begin{array}{cc}
d_{i j}^{22} & 2 \sigma d_{i j}^{21} \\
2 \sigma d_{i j}^{21} & d_{i j}^{11}
\end{array}\right)
$$

Due to the constraints (21) $-(22)$, the charge-spin correlations entering the matrix elements of (33) get exactly decoupled from each other, such that

$$
\begin{aligned}
& d_{i j}^{22}=\mathcal{K}_{22}\left(\chi_{i j}^{c}+\chi_{i j}^{S}\right)-\mathcal{K}_{11} \chi_{i j}^{s-h} \\
& d_{i j}^{11}=\mathcal{K}_{11}\left[\chi_{i j}^{c}+\left(\chi_{1}-\chi_{2}\right) \nu_{i j}+\chi_{i j}^{S}\right]-\mathcal{K}_{22} \chi_{i j}^{s-h} \\
& d_{i j}^{21}=\mathcal{K}_{21}\left[\left(\chi_{i j}^{c}-\chi_{2} \nu_{i j}\right)+\chi_{i j}^{S}\right]-\mathcal{K}_{21} \chi_{i j}^{s-h},
\end{aligned}
$$

with the three spin reversal invariant weighted boson-boson correlation functions representing respectively charge-charge (c), spin-spin (S), and singlet-hopping (s-h) correlations:

$$
\begin{aligned}
& \chi_{i j}^{\mathrm{c}}=\nu_{i j}\left\langle N_{i} N_{j}\right\rangle / 4, \\
& \chi_{i j}^{\mathrm{S}}=\nu_{i j}\left\langle\mathbf{S}_{i} \mathbf{S}_{j}\right\rangle \\
& \chi_{i j}^{\mathrm{s}-\mathrm{h}}=\nu_{i j}\left\langle X_{i}^{02} X_{j}^{20}\right\rangle
\end{aligned}
$$

In (35), $\mathbf{S}_{i}=\left(S_{i}^{z}, S_{i}^{\sigma}\right)$, with $S_{i}^{z}=\left(X_{i}^{\sigma \sigma}-X_{i}^{\bar{\sigma} \bar{\sigma}}\right) / 2$ and $S_{i}^{\sigma}=X_{i}^{\sigma \bar{\sigma}}$.

The anomalous hopping $2 \times 2$ matrix $\hat{\Delta}_{i j \sigma}$ has a very special form namely,

$$
\hat{\Delta}_{i j \sigma}=\left(\begin{array}{cc}
-\mathcal{K}_{21} \cdot 2 \sigma & \frac{1}{2}\left(\mathcal{K}_{11}+\mathcal{K}_{22}\right) \\
-\frac{1}{2}\left(\mathcal{K}_{11}+\mathcal{K}_{22}\right) & \mathcal{K}_{21} \cdot 2 \sigma
\end{array}\right) \chi_{i j}^{\text {pair }}
$$

where the spin reversal invariant weighted boson-boson pairing (pair) correlation function is given by

$$
\begin{aligned}
\chi_{i j}^{\text {pair }} & =\nu_{i j}\left\langle X_{i}^{02} N_{j}\right\rangle=2 \nu_{i j}\left\langle X_{i}^{02}\left(X_{j}^{\sigma \sigma}+X_{j}^{22}\right)\right\rangle= \\
& =-\nu_{i j}\left\langle N_{j}^{h} X_{i}^{02}\right\rangle=-2 \nu_{i j}\left\langle\left(X_{j}^{\sigma \sigma}+X_{j}^{00}\right) X_{i}^{02}\right\rangle .
\end{aligned}
$$


In Eqs. (38) and (39), the derivation of the second expression from the first one makes use of the spin reversal invariance property (23).

To get a workable expression of the frequency matrix, approximations have to be derived for the boson-boson statistical averages entering the two-site hopping matrix elements. In the next section we show that the method of reference [12, yielding the pairing correlation function $\left\langle X_{i}^{02} N_{j}\right\rangle$ in terms of GMFA Green functions within an approach able to identify and rule out exponentially small terms, can be extended to the singlet hopping correlations $\left\langle X_{i}^{02} X_{j}^{20}\right\rangle$ as well.

\section{Hopping processes involving singlets}

The right approach to the reduction of the order of correlation of the boson-boson statistical averages $\left\langle X_{i}^{02} X_{j}^{\lambda \mu}\right\rangle=\left\langle X_{j}^{\lambda \mu} X_{i}^{02}\right\rangle$ goes differently for the hole-doped and electron-doped cuprates.

\section{- Reduction of the correlation order for hole-doped cuprates}

In these systems, the Fermi level (the zero point energy) stays in the singlet subband. We get the estimates $E_{2} \simeq-\Delta, E_{2}-\Delta \simeq-2 \Delta, E_{2}+\Delta \simeq 0$. With $\Delta \sim 3 \mathrm{eV}, \beta \Delta \sim 3.5 \cdot 10^{4} \mathrm{~T}^{-1}$. Therefore, at $T \lesssim 300 \mathrm{~K}$, the quantities containing the factor $\mathrm{e}^{\beta E_{2}} \simeq \mathrm{e}^{-\beta \Delta} \lesssim \mathrm{e}^{-100}<10^{-44}$ are negligible.

We start with the following form of the spectral theorem [9]

$$
\left\langle X_{i}^{02} X_{j}^{\lambda \mu}\right\rangle=\frac{\mathrm{i}}{2 \pi} \int_{-\infty}^{+\infty} \frac{\mathrm{d} \omega}{1+\mathrm{e}^{-\beta \omega}}\left[\left\langle\left\langle X_{i}^{02} \mid X_{j}^{\lambda \mu}\right\rangle\right\rangle_{\omega+\mathrm{i} \varepsilon}-\left\langle\left\langle X_{i}^{02} \mid X_{j}^{\lambda \mu}\right\rangle\right\rangle_{\omega-\mathrm{i} \varepsilon}\right],
$$

written for anticommutator retarded $(\omega+\mathrm{i} \varepsilon)$, respectively advanced $(\omega-\mathrm{i} \varepsilon)$ Green functions. Their equation of motion in the $(\mathbf{r}, \omega)$-representation is

$$
\left(\omega-E_{2}\right)\left\langle\left\langle X_{i}^{02} \mid X_{j}^{\lambda \mu}\right\rangle\right\rangle_{\omega} \simeq 2\left\langle X_{i}^{02} X_{j}^{\lambda \mu}\right\rangle+\mathcal{K}_{21} \sum_{\sigma} 2 \sigma\left[\left\langle\left\langle\tau_{1, i}^{0 \bar{\sigma}, 0 \sigma} \mid X_{j}^{\lambda \mu}\right\rangle\right\rangle_{\omega}-\left\langle\left\langle\tau_{1, i}^{\sigma 2, \bar{\sigma} 2} \mid X_{j}^{\lambda \mu}\right\rangle\right\rangle_{\omega}\right]
$$

where, for the sake of simplicity, the labels $\pm \mathrm{i} \varepsilon, \varepsilon=0^{+}$, describing respectively the retarded and the advanced Green functions have been omitted. In Eq. (41), the higher order r.h.s. contributions coming from the inband hopping terms have been dropped off. Replacing (41) in (40), we get

$$
\begin{aligned}
\left\langle X_{i}^{02} X_{j}^{\lambda \mu}\right\rangle \simeq & \mathcal{K}_{21} \sum_{\sigma} 2 \sigma \int_{-\infty}^{+\infty} \frac{\mathrm{d} \omega}{1+\mathrm{e}^{-\beta \omega}} \times \\
& \times\left(-\frac{1}{\pi}\right) \operatorname{Im}\left[\frac{1}{\omega-E_{2}+\mathrm{i} \varepsilon}\left(\left\langle\left\langle\tau_{1, i}^{0 \bar{\sigma}, 0 \sigma} \mid X_{j}^{\lambda \mu}\right\rangle\right\rangle_{\omega+\mathrm{i} \varepsilon}-\left\langle\left\langle\tau_{1, i}^{\sigma 2, \bar{\sigma} 2} \mid X_{j}^{\lambda \mu}\right\rangle\right\rangle_{\omega+\mathrm{i} \varepsilon}\right)\right] .
\end{aligned}
$$

To evaluate the imaginary part, we use the identity [9]

$$
\frac{1}{\omega-E_{2}+\mathrm{i} \varepsilon}=\mathcal{P} \frac{1}{\omega-E_{2}}-\mathrm{i} \pi \delta\left(\omega-E_{2}\right) .
$$

The integrals over the $\delta$-function yield (finite) GF real parts at $\omega=E_{2}$, multiplied by a thermodynamic factor $\sim \mathrm{e}^{-\beta \Delta} \ll 1$. The imaginary part of the hole subband GF 
$\left\langle\left\langle\tau_{1, i}^{0 \bar{\sigma}, 0 \sigma} \mid X_{j}^{\lambda \mu}\right\rangle\right\rangle_{\omega+\mathrm{i} \varepsilon}$ shows a $\delta$-like maximum at $\omega=E_{2}-\Delta$, where $\left(\omega-E_{2}\right)^{-1} \simeq \Delta^{-1}$ and the thermodynamic factor reaches a value $\sim \mathrm{e}^{-2 \Delta}$. The only non-negligible contribution to the principal part integral comes from the singlet subband GF $\left\langle\left\langle\tau_{1, i}^{\sigma 2, \bar{\sigma} 2} \mid X_{j}^{\lambda \mu}\right\rangle\right\rangle_{\omega+\mathrm{i} \varepsilon}$ the imaginary part of which shows a $\delta$-like maximum at $\omega=E_{2}+\Delta \simeq 0$. This allows us to approximate $\left(\omega-E_{2}\right)^{-1} \approx \Delta^{-1}$ within the integral over the singlet subband GF to get

$$
\left\langle X_{i}^{02} X_{j}^{\lambda \mu}\right\rangle \simeq\left(1-\delta_{i j}\right) \frac{\mathcal{K}_{21}}{\Delta} \sum_{\sigma} 2 \bar{\sigma}\left\langle\tau_{1, i}^{\sigma 2, \bar{\sigma} 2} X_{j}^{\lambda \mu}\right\rangle
$$

Replacing this result in Eq. (38) and using (21) we get

$$
\chi_{i j}^{\text {pair }} \simeq\left(1-\delta_{i j}\right) \frac{\mathcal{K}_{21} \nu_{i j}}{\Delta}\left[4 \nu_{i j} \cdot 2 \bar{\sigma}\left\langle X_{i}^{\sigma 2} X_{j}^{\bar{\sigma} 2}\right\rangle-\sum_{m \neq(i, j)} \nu_{i m} \sum_{\sigma} 2 \sigma\left\langle X_{i}^{\sigma 2} X_{m}^{\bar{\sigma} 2} N_{j}\right\rangle\right]
$$

Omitting the three-site terms, we get the two-site approximation of the superconducting pairing originating in the singlet subband,

$$
\chi_{i j}^{\text {pair }} \simeq\left(1-\delta_{i j}\right) \frac{4 \mathcal{K}_{21} \nu_{i j}^{2}}{\Delta} \cdot 2 \bar{\sigma}\left\langle X_{i}^{\sigma 2} X_{j}^{\bar{\sigma} 2}\right\rangle,
$$

which reproduces the well-known two-site exchange term of the $t$ - $J$ model.

For the singlet hopping correlation function, (42) yields the two-site approximation

$$
\chi_{i j}^{s-h} \simeq\left(1-\delta_{i j}\right) \frac{2 \mathcal{K}_{21} \nu_{i j}^{2}}{\Delta} \cdot 2 \bar{\sigma}\left\langle X_{i}^{\sigma 2} X_{j}^{\bar{\sigma} 0}\right\rangle
$$

\section{- Reduction of the correlation order for electron-doped cuprates}

The Fermi level (the zero point energy) stays now in the hole subband. We have the estimates $E_{2} \simeq \Delta, E_{2}+\Delta \simeq 2 \Delta, E_{2}-\Delta \simeq 0$.

It is convenient now to start with the alternative form of the spectral theorem [9]

$$
\left\langle X_{j}^{\lambda \mu} X_{i}^{02}\right\rangle=\frac{\mathrm{i}}{2 \pi} \int_{-\infty}^{+\infty} \frac{\mathrm{d} \omega}{\mathrm{e}^{\beta \omega}+1}\left[\left\langle\left\langle X_{i}^{02} \mid X_{j}^{\lambda \mu}\right\rangle\right\rangle_{\omega+\mathrm{i} \varepsilon}-\left\langle\left\langle X_{i}^{02} \mid X_{j}^{\lambda \mu}\right\rangle\right\rangle_{\omega-\mathrm{i} \varepsilon}\right],
$$

with the retarded and advanced GFs following from the same equation (41).

Exponentially small quantities result from the $\delta$-term of $\left(\omega-E_{2}+\mathrm{i} \varepsilon\right)^{-1}$ and from the singlet subband GF $\left\langle\left\langle\tau_{1, i}^{\sigma 2, \bar{\sigma}} \mid X_{j}^{\lambda \mu}\right\rangle\right\rangle_{\omega+\mathrm{i} \varepsilon}$. The hole subband GF $\left\langle\left\langle\tau_{1, i}^{0 \bar{\sigma}, 0 \sigma} \mid X_{j}^{\lambda \mu}\right\rangle\right\rangle_{\omega+\mathrm{i} \varepsilon}$, yields the non-negligible contribution

$$
\left\langle X_{j}^{\lambda \mu} X_{i}^{02}\right\rangle \simeq\left(1-\delta_{i j}\right) \frac{\mathcal{K}_{21}}{\Delta} \sum_{\sigma} 2 \bar{\sigma}\left\langle X_{j}^{\lambda \mu} \tau_{1, i}^{0 \bar{\sigma}, 0 \sigma}\right\rangle
$$

Replacing in (39) and omitting the three-site terms, we get the two-site approximation of the superconducting pairing originating in the hole subband,

$$
\chi_{i j}^{\text {pair }} \simeq\left(1-\delta_{i j}\right) \frac{4 \mathcal{K}_{21} \nu_{i j}^{2}}{\Delta} \cdot 2 \sigma\left\langle X_{i}^{0 \bar{\sigma}} X_{j}^{0 \sigma}\right\rangle
$$

Finally, the two-site approximation of the singlet-hopping correlation function is

$$
\left\langle X_{i}^{02} X_{j}^{20}\right\rangle \simeq\left(1-\delta_{i j}\right) \frac{2 \mathcal{K}_{21} \nu_{i j}^{2}}{\Delta} \cdot 2 \bar{\sigma}\left\langle X_{i}^{0 \bar{\sigma}} X_{j}^{2 \sigma}\right\rangle .
$$

In conclusion, the GMFA superconducting pairing is a second order effect. The lowest order contribution to it originates in interband hopping correlating annihilation 
(or creation) of pairs of spins at neighbouring lattice sites $i$ and $j$ within that energy subband which crosses the Fermi level.

Similarly, the singlet hopping is a second order effect as well. It mainly proceeds by interband $i \rightleftarrows j$ single particle jumps from the upper energy subband to the lower energy subband.

\section{Frequency matrix in $(\mathbf{q}, \omega)$-representation}

The calculation of the matrix elements of $\tilde{\mathcal{A}}_{\sigma}(\mathbf{q})$ from Eq. (9) asks for three essentially different kinds of Fourier transforms, namely,

- The averages of the Hubbard 1-forms entering Eqs. (31) and (32) result in sums of products of q-space averages and geometrical form factors:

$$
\left\langle\tau_{1, i}^{\lambda \mu, \nu \varphi}\right\rangle=\sum_{\alpha=1}^{3} \nu_{\alpha} \cdot \frac{1}{N} \sum_{\mathbf{q}}\left\langle X^{\lambda \mu} X^{\nu \varphi}\right\rangle_{\mathbf{q}} \gamma_{\alpha}(\mathbf{q})
$$

for label sets $\{(\lambda \mu, \nu \varphi)\} \in\{(0 \bar{\sigma}, \bar{\sigma} 0) ;(\sigma 2,2 \sigma) ;(\sigma 2, \bar{\sigma} 0)\}$.

The quantity $\left\langle X^{\lambda \mu} X^{\nu \varphi}\right\rangle_{\mathbf{q}}$ denotes the average of the $\mathbf{q}$-space image of the product of Hubbard operators of labels $\lambda \mu$ and $\nu \varphi$ respectively,

$$
\left\langle X^{\lambda \mu} X^{\nu \varphi}\right\rangle_{\mathbf{q}}=\frac{\mathrm{i}}{2 \pi} \int_{-\infty}^{+\infty} \frac{\mathrm{d} \omega}{1+\mathrm{e}^{-\beta \omega}}\left[G^{\lambda \mu, \nu \varphi}(\mathbf{q}, \omega+\mathrm{i} \varepsilon)-G^{\lambda \mu, \nu \varphi}(\mathbf{q}, \omega-\mathrm{i} \varepsilon)\right]
$$

Finally, in Eq. (50), $\gamma_{\alpha}(\mathbf{q})$ denote the $\mathrm{nn}(\alpha=1)$, nnn $(\alpha=2)$, and third neighbour $(\alpha=3)$ geometrical form factors, $\gamma_{1}(\mathbf{q})=2\left[\cos \left(q_{x} a_{x}\right)+\cos \left(q_{y} a_{y}\right)\right]$, $\gamma_{2}(\mathbf{q})=4 \cos \left(q_{x} a_{x}\right) \cos \left(q_{y} a_{y}\right), \gamma_{3}(\mathbf{q})=2\left[\cos \left(2 q_{x} a_{x}\right)+\cos \left(2 q_{y} a_{y}\right)\right]$.

- For the two-site weighted singlet hopping (36) and the superconducting pairing (38), the Fourier transforms result in convolutions of specific averages and geometrical form factors. The results are as follows:

- Singlet hopping

$$
\chi^{\mathrm{s}-\mathrm{h}}(\mathbf{q})=\sum_{\alpha=1}^{3} \nu_{\alpha}^{2} \cdot \frac{1}{N} \sum_{\mathbf{k}} \Xi_{\mathbf{k}} \gamma_{\alpha}(\mathbf{q}-\mathbf{k})
$$

where $\Xi_{\mathbf{k}}=2 \sigma\left\langle X^{\sigma 2} X^{\bar{\sigma} 0}\right\rangle_{\mathbf{k}}$, while $\Xi_{\mathbf{k}}=2 \sigma\left\langle X^{0 \bar{\sigma}} X^{2 \sigma}\right\rangle_{\mathbf{k}}$ for hole-doped and electrondoped cuprates respectively, with averages defined in (51).

- Superconducting pairing

$$
\chi^{\text {pair }}(\mathbf{q})=\sum_{\alpha=1}^{3} \nu_{\alpha}^{2} \cdot \frac{1}{N} \sum_{\mathbf{k}} \Pi_{\mathbf{k}} \gamma_{\alpha}(\mathbf{q}-\mathbf{k})
$$

where $\Pi_{\mathbf{k}}=2 \bar{\sigma}\left\langle X^{\sigma 2} X^{\bar{\sigma} 2}\right\rangle_{\mathbf{k}}$, while $\Pi_{\mathbf{k}}=2 \sigma\left\langle X^{0 \bar{\sigma}} X^{0 \sigma}\right\rangle_{\mathbf{k}}$ for hole-doped and electrondoped cuprates respectively, with averages defined in (51).

- The charge-charge and spin-spin correlation functions (34) and (35) are treated approximately following [8, 10]: 
- The order of the charge-charge correlation function $\left\langle N_{i} N_{j}\right\rangle$ is lowered using a Hubbard type I approximation decoupling procedure $\left\langle N_{i} N_{j}\right\rangle \simeq\left\langle N_{i}\right\rangle\left\langle N_{j}\right\rangle=2 \chi_{2}$.

- The spin-spin correlation function $\left\langle\mathbf{S}_{i} \mathbf{S}_{j}\right\rangle$ is kept undecoupled, but treated phenomenologically. Eq. (2) implies the occurrence of up to three non-vanishing spin-spin correlation functions: $\mathrm{nn}, \chi_{1}^{S}=\left\langle S_{i} S_{i \pm a_{x / y}}\right\rangle, \mathrm{nnn}, \chi_{2}^{S}=\left\langle S_{i} S_{i \pm a_{x} \pm a_{y}}\right\rangle$, and $\chi_{3}^{S}=\left\langle S_{i} S_{i \pm 2 a_{x / y}}\right\rangle$. These are site independent quantities.

Using the above results, we get from (9) and (27) the mathematical structure of the frequency matrix $\tilde{\mathcal{A}}_{\sigma}(\mathbf{q})$ as follows,

$$
\tilde{\mathcal{A}}_{\sigma}(\mathbf{q})=\left(\begin{array}{cc}
\hat{E}_{\sigma}(\mathbf{q}) & \hat{\Phi}_{\sigma}(\mathbf{q}) \\
\left(\hat{\Phi}_{\sigma}(\mathbf{q})\right)^{\dagger} & -\left(\hat{E}_{\bar{\sigma}}(\mathbf{q})\right)^{\top}
\end{array}\right) .
$$

The normal $2 \times 2$ matrix contributions to $\tilde{\mathcal{A}}_{\sigma}(\mathbf{q})$ show the characteristic $\sigma$-dependence,

$$
\hat{E}_{\sigma}(\mathbf{q})=\left(\begin{array}{cc}
c_{22} & 2 \sigma c_{21} \\
2 \sigma c_{21}^{*} & c_{11}
\end{array}\right) ; \quad-\left(\hat{E}_{\bar{\sigma}}(\mathbf{q})\right)^{\top}=\left(\begin{array}{cc}
-c_{22} & 2 \sigma c_{21}^{*} \\
2 \sigma c_{21} & -c_{11}
\end{array}\right)
$$

with the $\sigma$-independent terms $c_{a b}$ carrying normal one-site and two-site matrix elements,

$$
\begin{aligned}
c_{22} & \equiv c_{22}(\mathbf{q})=\left(E_{1}+\Delta\right) \chi_{2}+a_{22}+d_{22}(\mathbf{q}) \\
c_{11} & \equiv c_{11}(\mathbf{q})=E_{1} \chi_{1}+a_{22}+d_{11}(\mathbf{q}) \\
c_{21} & \equiv c_{21}(\mathbf{q})=a_{21}+d_{21}(\mathbf{q}) \\
d_{a b}(\mathbf{q}) & =\mathcal{K}_{a b} \sum_{\alpha=1}^{3} \nu_{\alpha} \gamma_{\alpha}(\mathbf{q})\left[\chi_{\alpha}^{S}+(-1)^{a+b} \chi_{a} \chi_{b}\right]+\frac{1}{2} J_{a b} \chi^{\mathrm{s}-\mathrm{h}}(\mathbf{q})
\end{aligned}
$$

The one-site terms are defined by Eqs. (31)-(32) and (50). The exchange energy parameters are given by

$$
J_{a b}=4 \mathcal{K}_{a b} \mathcal{K}_{21} / \Delta, \quad\{a b\} \in\{22,11,21\},
$$

while the singlet hopping contribution $\chi^{\mathrm{s}-\mathrm{h}}(\mathbf{q})$ is given by Eq. (52).

The anomalous $2 \times 2$ matrix contributions to $\tilde{\mathcal{A}}_{\sigma}(\mathbf{q})$, obtained from (37), show the characteristic $\sigma$-dependence,

$$
\hat{\Phi}_{\sigma}(\mathbf{q})=\left(\begin{array}{cc}
-2 \sigma \xi_{1} b & \xi_{2} b \\
-\xi_{2} b & 2 \sigma \xi_{1} b
\end{array}\right) ; \quad\left(\hat{\Phi}_{\sigma}(\mathbf{q})\right)^{\dagger}=\left(\begin{array}{cc}
-2 \sigma \xi_{1} b^{*} & -\xi_{2} b^{*} \\
\xi_{2} b^{*} & 2 \sigma \xi_{1} b^{*}
\end{array}\right)
$$

with $\xi_{1}=J_{21}, \xi_{2}=\left(J_{11}+J_{22}\right) / 2$, whereas $b \equiv b(\mathbf{q})$ is a shorthand notation for the pairing matrix element (53) .

Remark 1 The spin reversal $\sigma \rightarrow \bar{\sigma}$ symmetry properties of the elemental Green functions entering the matrix GF (3) are identical to those established for the underlying frequency matrix $\tilde{\mathcal{A}}_{\sigma}(\mathbf{q})$. 


\section{GMFA Green function}

From Eqs. (15) and (18) it follows that the matrix $\tilde{\chi}$, Eq. (8) , is diagonal and spin reversal invariant, with two nonvanishing matrix elements,

$$
\tilde{\chi}=\left(\begin{array}{cc}
\hat{\chi} & \hat{0} \\
\hat{0} & \hat{\chi}
\end{array}\right), \quad \hat{\chi}=\left(\begin{array}{cc}
\chi_{2} & 0 \\
0 & \chi_{1}
\end{array}\right), \quad \hat{0}=\left(\begin{array}{ll}
0 & 0 \\
0 & 0
\end{array}\right),
$$

where $\chi_{2}$ and $\chi_{1}$ are given by Eqs. (29) and (30) respectively.

Replacing in (7) the expressions (58) of the matrix $\tilde{\chi}$ and (54) of the frequency matrix $\tilde{\mathcal{A}}_{\sigma}(\mathbf{q})$, we get a structure of the GMFA-GF matrix obeying the general symmetry properties established in [11],

$$
\tilde{G}_{\sigma}^{0}(\mathbf{q}, \omega)=\left(\begin{array}{cc}
\hat{G}_{\sigma}^{0}(\mathbf{q}, \omega) & \hat{F}_{\sigma}^{0}(\mathbf{q}, \omega) \\
\left(\hat{F}_{\sigma}^{0}(\mathbf{q}, \omega)\right)^{\dagger} & -\left(\hat{G}_{\bar{\sigma}}^{0}(\mathbf{q},-\omega)\right)^{\top}
\end{array}\right),
$$

where the argument $\omega$ carries, in fact, the complex value $\omega+i \varepsilon, \varepsilon=0^{+}$. (Hence the elemental GFs containing the argument $\omega$ point to retarded GFs, while those containing the argument $-\omega$ point to advanced GFs.)

The normal $2 \times 2$ matrix $\hat{G}_{\sigma}^{0}(\mathbf{q}, \omega)$ shows the characteristic $\sigma$-dependence,

$$
\hat{G}_{\sigma}^{0}(\mathbf{q}, \omega)=\left(\begin{array}{cc}
g_{22}(\mathbf{q}, \omega) & 2 \sigma g_{21}(\mathbf{q}, \omega) \\
2 \sigma g_{21}^{*}(\mathbf{q}, \omega) & g_{11}(\mathbf{q}, \omega)
\end{array}\right) \cdot \frac{1}{\mathcal{D}(\mathbf{q}, \omega)}
$$

with the $\sigma$-independent components $g_{a b}(\mathbf{q}, \omega)$ found from

$$
g_{a b}(\mathbf{q}, \omega)=A_{a b} \omega^{3}+B_{a b} \omega^{2}+C_{a b} \omega+D_{a b}, \quad\{a b\} \in\{22,11,21\} .
$$

Here the coefficients $A_{a b}$ are given respectively by

$$
A_{22}=\chi_{2}, \quad A_{11}=\chi_{1}, \quad A_{21}=0
$$

while $B_{a b}, C_{a b}, D_{a b}$ are $\mathbf{q}$-dependent coefficients:

$$
\begin{gathered}
B_{22}(\mathbf{q})=c_{22}, \quad B_{11}(\mathbf{q})=c_{11}, \quad B_{21}(\mathbf{q})=c_{21} \\
C_{22}(\mathbf{q})=-\left[\chi_{2}\left(c_{11}^{2}+\xi_{1}^{2}|b|^{2}\right)+\chi_{1}\left(\left|c_{21}\right|^{2}+\xi_{2}^{2}|b|^{2}\right)\right] / \chi_{1}^{2} \\
C_{11}(\mathbf{q})=-\left[\chi_{1}\left(c_{22}^{2}+\xi_{1}^{2}|b|^{2}\right)+\chi_{2}\left(\left|c_{21}\right|^{2}+\xi_{2}^{2}|b|^{2}\right)\right] / \chi_{2}^{2} \\
C_{21}(\mathbf{q})=\left[c_{21}\left(\chi_{2} c_{11}+\chi_{1} c_{22}\right)-\xi_{1} \xi_{2}|b|^{2}\right] /\left(\chi_{1} \chi_{2}\right) \\
D_{22}(\mathbf{q})=-\left[c_{11}\left(c_{22} c_{11}-\left|c_{21}\right|^{2}\right)+\left(c_{22} \xi_{1}^{2}+c_{11} \xi_{2}^{2}+2 \Re\left(c_{21}\right) \xi_{1} \xi_{2}\right)|b|^{2}\right] / \chi_{1}^{2} \\
D_{11}(\mathbf{q})=-\left[c_{22}\left(c_{22} c_{11}-\left|c_{21}\right|^{2}\right)+\left(c_{11} \xi_{1}^{2}+c_{22} \xi_{2}^{2}+2 \Re\left(c_{21}\right) \xi_{1} \xi_{2}\right)|b|^{2}\right] / \chi_{2}^{2} \\
D_{21}(\mathbf{q})=\left\{c_{21}\left(c_{22} c_{11}-\left|c_{21}\right|^{2}\right)-\left[c_{21}^{*} \xi_{1}^{2}+c_{21} \xi_{2}^{2}+\left(c_{22}+c_{11}\right) \xi_{1} \xi_{2}\right]|b|^{2}\right\} /\left(\chi_{1} \chi_{2}\right)
\end{gathered}
$$

The anomalous $2 \times 2$ matrix $\hat{F}_{\sigma}^{0}(\mathbf{q}, \omega)$ shows the characteristic $\sigma$-dependence,

$$
\hat{F}_{\sigma}^{0}(\mathbf{q}, \omega)=\left(\begin{array}{cc}
2 \sigma f_{22}(\mathbf{q}, \omega) & f_{21}(\mathbf{q}, \omega) \\
-f_{21}(\mathbf{q},-\omega) & 2 \sigma f_{11}(\mathbf{q}, \omega)
\end{array}\right) \cdot \frac{1}{\mathcal{D}(\mathbf{q}, \omega)}
$$


with the elemental GFs $f_{a b}(\mathbf{q}, \omega)$ given by

$$
\begin{aligned}
& f_{a a}(\mathbf{q}, \omega)=\left(P_{a a} \omega^{2}+R_{a a}\right) b, \quad\{a a\} \in\{22,11\}, \\
& f_{21}(\mathbf{q}, \omega)=\left(P_{21} \omega^{2}+Q_{21} \omega+R_{21}\right) b .
\end{aligned}
$$

Here, $P_{22}=-\xi_{1}, P_{11}=\xi_{1}$, and $P_{21}=-\xi_{2}$ are $\mathbf{q}$-independent, while

$$
\begin{aligned}
& R_{22}(\mathbf{q})=\left[\left(c_{11}^{2}+c_{21}^{2}\right) \xi_{1}+2 c_{11} c_{21} \xi_{2}+\xi_{1}\left(\xi_{1}^{2}-\xi_{2}^{2}\right)|b|^{2}\right] / \chi_{1}^{2} \\
& R_{11}(\mathbf{q})=-\left[\left(c_{22}^{2}+c_{21}^{*}\right) \xi_{1}+2 c_{22} c_{21}^{*} \xi_{2}+\xi_{1}\left(\xi_{1}^{2}-\xi_{2}^{2}\right)|b|^{2}\right] / \chi_{2}^{2} \\
& R_{21}(\mathbf{q})=\left[\left(c_{11} c_{21}^{*}+c_{22} c_{21}\right) \xi_{1}+\left(c_{22} c_{11}+\left|c_{21}\right|^{2}\right) \xi_{2}-\xi_{2}\left(\xi_{1}^{2}-\xi_{2}^{2}\right)|b|^{2}\right] /\left(\chi_{1} \chi_{2}\right) \\
& Q_{21}(\mathbf{q})=\left[\left(\chi_{2} c_{21}-\chi_{1} c_{21}^{*}\right) \xi_{1}+\left(\chi_{2} c_{11}-\chi_{1} c_{22}\right) \xi_{2}\right] /\left(\chi_{1} \chi_{2}\right) .
\end{aligned}
$$

The denominator $\mathcal{D}(\mathbf{q}, \omega)$ occurring in Eqs. (60) and (61), which is proportional to the determinant of the matrix $\tilde{\chi} \omega-\tilde{A}_{\sigma}(\mathbf{q})$ in (7), shows the following monic bi-quadratic dependence in $\omega$ :

$$
\mathcal{D}(\mathbf{q}, \omega)=\left(\omega^{2}-u \omega+v\right)\left(\omega^{2}+u \omega+v\right),
$$

where $v=v(\mathbf{q})$ and $u=u(\mathbf{q})$ are found respectively from

$$
\begin{aligned}
v^{2}= & \left\{\left[\left(c_{22} c_{11}-\left|c_{21}\right|^{2}\right)-\left(\xi_{1}^{2}-\xi_{2}^{2}\right)|b|^{2}\right]^{2}+\left[\left[\left(c_{22}+c_{11}\right)+2 \Re\left(c_{21}\right)\right]^{2} \xi_{1}^{2}-\right.\right. \\
& \left.\left.-4\left(c_{22}+c_{11}\right) \Re\left(c_{21}\right) \xi_{1}\left(\xi_{1}-\xi_{2}\right)-4\left|c_{21}\right|^{2}\left(\xi_{1}^{2}-\xi_{2}^{2}\right)\right]|b|^{2}\right\} /\left(\chi_{1}^{2} \chi_{2}^{2}\right) \\
u^{2}- & 2 v=\frac{1}{\chi_{1}^{2}}\left(c_{11}^{2}+\xi_{1}^{2}|b|^{2}\right)+\frac{1}{\chi_{2}^{2}}\left(c_{22}^{2}+\xi_{1}^{2}|b|^{2}\right)+\frac{2}{\chi_{1} \chi_{2}}\left(\left|c_{21}\right|^{2}+\xi_{2}^{2}|b|^{2}\right) .
\end{aligned}
$$

A necessary consistency condition to be satisfied by the parameters of the model at any vector $\mathbf{q}$ inside the Brillouin zone is $v^{2}(\mathbf{q}) \geq 0$.

Remark 2 The zeros of the determinant of the GMFA-GF,

$$
\mathcal{D}(\mathbf{q}, \omega)=0
$$

provide the GMFA energy spectrum of the system.

At every wave vector $\mathbf{q}$ inside the Brillouin zone, this yields for the superconducting state the energy eigenvalue set

$$
\begin{aligned}
& \left\{\Omega_{1}(\mathbf{q}), \Omega_{2}(\mathbf{q}),-\Omega_{2}(\mathbf{q}),-\Omega_{1}(\mathbf{q})\right\} \\
& \Omega_{1,2}(\mathbf{q})=(u / 2) \pm \sqrt{(u / 2)^{2}-v}
\end{aligned}
$$

In the normal state $(b=0)$, Eqs. (63) and (64) reduce respectively to

$$
\begin{aligned}
& v_{0}=\left(c_{22} / \chi_{2}\right)\left(c_{11} / \chi_{1}\right)-\left|c_{21}\right|^{2} /\left(\chi_{1} \chi_{2}\right) \\
& u_{0}=\left(c_{22} / \chi_{2}\right)+\left(c_{11} / \chi_{1}\right)
\end{aligned}
$$

such that the energy spectrum is given by the roots of the second order equation $\omega^{2}-u_{0} \omega+v_{0}=0$ solved in [8].

Finally, if we assume a pure Hubbard model (i.e., energy band independent hopping parameters, $\mathcal{K}_{11}=\mathcal{K}_{22}=\mathcal{K}_{21} \equiv t$, [10]), then a significant simplification of the equations derived in the last two sections is obtained. The normal $2 \times 2$ matrix $\hat{E}_{\sigma}(\mathbf{q})$ 
becomes symmetric and so is the normal GMFA-GF $\hat{G}_{\sigma}^{0}(\mathbf{q}, \omega)$. Moreover, there is a single exchange energy parameter in (57), $\xi_{1}=\xi_{2} \equiv J=4 t^{2} / \Delta$, which simplifies the

anomalous $2 \times 2$ frequency matrix to $\hat{\Phi}_{\sigma}(\mathbf{q})=\left(\begin{array}{cc}2 \sigma & 1 \\ -1 & 2 \sigma\end{array}\right) J b$, such that the quantities $u$ and $v$ in the expression (62) of the GF determinant reduce to

$$
\begin{aligned}
& v^{2}=\left[\left(c_{22} c_{11}-c_{21}^{2}\right)^{2}+\left(c_{22}+c_{11}+2 c_{21}\right)^{2} J^{2}|b|^{2}\right] /\left(\chi_{1}^{2} \chi_{2}^{2}\right) \\
& u^{2}-2 v=\left[\chi_{2}^{2} c_{11}^{2}+\chi_{1}^{2} c_{22}^{2}+2 \chi_{1} \chi_{2} c_{21}^{2}+J^{2}|b|^{2}\right] /\left(\chi_{1}^{2} \chi_{2}^{2}\right) .
\end{aligned}
$$

A non-negative value $v \geq 0$ always follows from Eq. (67), however, the reality of the solutions (66) needs investigation of the domain of variation of the adjustable parameters of the model.

\section{Conclusions}

The two-band Hubbard model of the high $T_{c}$ superconductivity in cuprates [8, 12] uses Hubbard operator algebra on a physical system characterized by specific invariance symmetries with respect to translations and spin reversal.

In the present paper we have shown that the system symmetries result either in invariance properties or exact vanishing of several characteristic statistical averages. The vanishing of the one-site anomalous matrix elements is shown to be a property which is embedded in the Hubbard operator algebra. Another worth mentioning consequence following from the spin reversal invariance properties of the two-site statistical averages is the exact decoupling from each other of the charge and spin correlations entering the matrix elements of the frequency matrix. The use of these results allowed rigorous derivation and simplification of the expression of the frequency matrix of the generalized mean field approximation (GMFA) Green function (GF) matrix of the model.

For the higher order boson-boson averages $\left\langle X_{i}^{02} X_{j}^{20}\right\rangle$ and $\left\langle X_{i}^{02} N_{j}\right\rangle$, which enter respectively the normal singlet hopping and anomalous exchange pairing contributions to the frequency matrix, an approximation procedure resulting in GMFA-GF expressions was described. The procedure avoids the current decoupling schemes [14, 15]. Its principle, first formulated in [12], consists in the identification and elimination of exponentially small contributions to the spectral theorem representations of these statistical averages.

A point worth noting is that the proper identification of exponentially small quantities asks for the use of different starting expressions of the spectral theorem for the hole-doped and electron-doped cuprates.

The results of the reduction procedure may be summarized as follows:

- The singlet hopping is a second order effect which may be described as interband $i \rightleftarrows j$ single particle jumps from the upper to the lower energy subband.

- The GMFA superconducting pairing is a second order effect, the lowest order contribution to which originates in interband hopping correlating the annihilation 
(creation) of spin pairs at neighbouring lattice sites $i$ and $j$ within that energy subband which crosses the Fermi level.

The derivation of the most general and simplest possible expressions of the frequency matrix and of the GMFA-GF matrix in the $(\mathbf{q}, \omega)$-representation enables reliable numerical investigation of the consequences coming from the adjustable parameters of the model (the degree of hole/electron doping, the energy gap $\Delta$, the hopping parameters).

Another open question of the GF approach to the solution of the present model is the use of the Hubbard operator algebra to get rigorous derivation and simplification of the Dyson equation of the complete Green function. As shown previously in [12], the self-energy corrections induce a spin fluctuation $d$-wave pairing originating in kinematic interaction in the second order.

These investigations are underway and results will be reported in a forthcoming paper.

\section{Acknowledgments}

The authors would like to express their gratitude to Prof. N.M. Plakida for useful advice and critical reading of the manuscript. Partial financial support was secured by the Romanian Authority for Scientific Research (Project 11404/31.10.2005 - SIMFAP).

\section{References}

[1] Damascelli A, Hussain Z and Shen Z -X 1986 Rev. Mod. Phys. 75473

[2] F.C. Zhang F C and T.M. Rice T M 1988 Phys. Rev. B 373759

[3] Emery V J 1987 Phys. Rev. Lett. 58 2794; Varma C M Schmitt-Rink S, and Abrahams E 1987 Solid State Commun. 62681

[4] Feiner L J, Jefferson J H and Raimondi R 1996 Phys. Rev. B 538751

[5] Yushankhai V Yu, Oudovenko V S and Hayn R 1997 Phys. Rev. B 5515562

[6] Plakida N M and Oudovenko V S 1999 Phys. Rev. B 59, 11949

[7] Plakida N M 2001 JETP Lett. 7436

[8] Plakida N M, Hayn R, and Richard J -L 1995 Phys. Rev. B 5116599

[9] Zubarev D N 1960 Sov. Phys. Usp. 3320

[10] Plakida N M and Oudovenko V S 2007 JETP 104230

[11] Plakida N M 1997 Physica C 282-287 1737

[12] Plakida N M, Anton L, Adam S, and Adam Gh 2003 ZhETF 124, 367; English transl.: 2003 JETP 97331

[13] Plakida N M 2006 Fiz. Nizkikh Temp. 32483

[14] Roth L M 1969 Phys. Rev. 184451

[15] Beenen J and Edwards D M 1995 Phys. Rev. B 52 13636; Avella A, Mancini F, Villani D and Matsumoto H 1997 Physica C 282-287 1757; Di Matteo T, Mancini F, Matsumoto H and Oudovenko V S 1997 Physica B 230-232 915; Stanescu T D, Martin I and Phillips Ph, 2000 Phys. Rev. B 624300 